\title{
Evolutionary robotics
}

\author{
Evert Haasdijk $\cdot$ Nicolas Bredeche $\cdot$ \\ Stefano Nolfi $\cdot$ A. E. Eiben
}

Received: 8 July 2014 / Accepted: 16 July 2014/Published online: 8 August 2014

(C) Springer-Verlag Berlin Heidelberg 2014

Evolutionary robotics combines evolutionary computing with robotics [1, 2, 4, 7-10]. It is a field that "aims to apply evolutionary computation techniques to evolve the overall design or controllers, or both, for real and simulated autonomous robots" [9]. It is a useful approach "both for investigating the design space of robotic applications and for testing scientific hypotheses of biological mechanisms and processes" [4].

Over the last fifteen years, evolutionary robotics has developed from the novel field described in Nolfi and Floreano's seminal book [7] to a more mature discipline. Evolutionary robotics is now also used to tackle problems involving complex dynamical systems, such as swarm robotics, modular robotics, flying and underwater robotics. Major research challenges have been identified and are currently being investigated by the community, ranging

E. Haasdijk · A. E. Eiben

VU University Amsterdam, Amsterdam, The Netherlands e-mail: e.haasdijk@vu.nl

A. E. Eiben

e-mail: gusz@cs.vu.nl

N. Bredeche $(\bowtie)$

Sorbonne Universités, UPMC Univ Paris 06, UMR 7222, ISIR, 75005 Paris, France

e-mail: nicolas.bredeche@upmc.fr

N. Bredeche

CNRS, UMR 7222, ISIR, 75005 Paris, France

\section{S. Nolfi}

Istituto di Scienze e Tecnologie della Cognizione, Consiglio Nazionale delle Ricerche, Via San Martino della Battaglia 44, 00185 Rome, Italy

e-mail: stefano.nolfi@istc.cnr.it from the impact of selection pressure to embodied evolution and lifelong learning.

From the perspective of evolutionary computing, evolutionary robotics is a particular application area that is different from, say, combinatorial optimisation. Somewhat oversimplifying, the main challenge in solving optimisation problems with evolutionary algorithms is the ruggedness of the fitness landscape defined by the objective function. Evolutionary robotics applications face additional problems: one is the very indirect link between controllable design details and the target feature(s). Another particular issue is the great variety of conditions and requirements under which a solution has to hold. Unlike candidate solutions in 'simple' optimisation, robot phenotypes (controller, morphology, or both) cannot be directly evaluated. Rather, it is the robot's behaviour that needs to be observed and assessed. Thus, regular evolutionary computing implies a three levels of organization: genotype-phenotype-fitness, while in evolutionary robotics the process comprises of four levels of organization: genotype-phenotype-behaviour-fitness. Additionally, the behavior exhibited by a robot is not only the product of the robot morphology and controller but is the emergent result of the robot/environmental interactions [6]. Thus, the evaluation of a candidate solution necessarily require the evaluation of the behavior that arise by robots that are situated in the external environment and are allowed to "live" long enough to experience a large variety of environmental conditions. Last but not least, desirable robot behaviour is almost never defined by one single skill (except for pure research purposes). Consequently, the design of the fitness function is far from trivial [5]. ${ }^{1}$

\footnotetext{
${ }^{1}$ Parts of this introduction are based on Eiben [3].
} 
Evolutionary Robotics has been (re)gaining momentum in recent years as evidenced by the programmes of the conferences GECCO, EvoStar, PPSN, and ALIFE and initiatives such as a Specialty Section (a journal-in-ajournal) on Evolutionary Robotics featured in the new Frontiers journal on Robotics and AI. This special issue on evolutionary robotics aims to recognise this recent growth as well as to further encourage the development of the field.

Doncieux and Mouret provide a survey in "Beyond Black-Box Optimization: a Review of Selective Pressures for Evolutionary Robotics". The motivational observation is that Evolutionary Robotics is different from mainstream Evolutionary Computing in that the selective pressure, and, in particular, the fitness function, cannot be a pure userdefined black box. Therefore, selective pressures in evolutionary robotics form a research topic of their own. The literature is reviewed through a newly introduced taxonomy that distinguishes two categories: goal refiners, aimed at changing the definition of a good solution, and process helpers, designed to help the search process. The review shows that many task-agnostic process helpers have been proposed during the last years, advancing the field towards a fully automated robot behaviour design process.

O'Dowd, Studley, and Winfield report on "The Distributed Co-Evolution of an On-Board Simulator and Controller for Swarm Robot Behaviours". They describe a novel approach to improve the transference between simulation and reality of controllers that co-evolve with an onboard simulator without an explicit measurement to compare the two domains. They show that the variation of onboard simulators across many robots in the swarm can be competitively exploited. However, the results vary over different test scenarios. The results indicate that the approach is sensitive to whether the real behavioural performance of the robot is able to inform on the state real environment.

The paper "An evolutionary robotics approach for the distributed control of satellite formations" by Izzo, Simões and de Croon describes evolutionary robotics techniques in the context of space applications, in particular decentralised formation flying. They design a control scheme for the MIT SPHERES robotic platform, currently on board the International Space Station. The evolutionary method successfully produces homogeneous controllers that can plan for the acquisition and maintenance of any triangular formation with high precision.

The paper "Simultaneous versus incremental learning of multiple skills by modular robots" by Rossi and Eiben addresses the question whether it is better to learn multiple skills simultaneously (all-at-once) or incrementally (oneby-one). They conduct an experimental study with modular robots of various shapes and sizes that need to acquire three different but correlated skills using real-time on-board evolution as the learning method. The results indicate that the one-by-one strategy is more efficient and more stable than the all-at-once strategy.

Interestingly, two of the four papers in this issue employ on-line evolution where robots adapt in their task environment. This contrasts with the more traditional off-line scheme (optimise first, then deploy fixed controllers) prevalent in the majority of the current literature. We, the editors, believe that a largely unused potential exists in online evolution and hope that these two studies will inspire others to explore and exploit this potential.

It is also interesting to note that two of the four papers emphasise the importance of selective pressure: why should selective pressure be considered in any specific manner in evolutionary robotics; how should it be formulated and applied?

The contributions highlight that evolutionary robotics requires more than simply applying regular evolutionary computation to the robotics domain-it poses particular, challenging research problems and opportunities. We hope that this special issue encourages researchers to join or persevere this lively and interesting area of research.

\section{References}

1. Bongard J (2013) Evolutionary robotics. Commun ACM 56(8):74-85

2. Doncieux S, Mouret JB, Bredeche N, Padois V (2011) Evolutionary robotics: exploring new horizons. In: Doncieux S, Bredeche N, Mouret JB (eds) New horizons in evolutionary robotics, studies in computational intelligence, vol 341. Springer, Berlin, pp 3-25

3. Eiben AE (2014) In vivo veritas: towards the evolution of things. In: Proceedings of parallel problem solving from nature-PPSN XIII. Lecture notes in computer science, Springer, Berlin

4. Floreano D, Husbands P, Nolfi S (2008) Evolutionary robotics. In: Siciliano B, Khatib O (eds) Springer handbook of robotics, vol Part G.61. Springer, Berlin, pp 1423-1451

5. Nelson AL, Barlow GJ, Doitsidis L (2009) Fitness functions in evolutionary robotics: a survey and analysis. Robot Auton Syst 57(4):345-370. doi:10.1016/j.robot.2008.09.009

6. Nolfi S (2009) Behavior and cognition as a complex adaptive system: insights from robotic experiments. Philosophy of complex systems, handbook on foundational/philosophical issues for complex systems in science. Elsevier, Amsterdam

7. Nolfi S, Floreano D (2000) Evolutionary robotics: the biology, intelligence, and technology of self-organizing machines. MIT Press, Cambridge

8. Trianni V (2008) Evolutionary swarm robotics-evolving selforganising behaviours in groups of autonomous robots, studies in computational intelligence, vol 108. Springer, Berlin

9. Vargas P, Paolo ED, Harvey I, Husbands P (eds) (2014) The horizons of evolutionary robotics. MIT Press, Cambridge

10. Wang L, Tan K, Chew C (2006) Evolutionary robotics: from algorithms to implementations. World Scientific Series in Robotics and Intelligent Systems, vol 28. World Scientific, Singapore 